\title{
Caracterización y Atención Temprana del Síndrome de Sotos
}

\section{Characterization and Early Warning of Sotos Syndrome}

\author{
Pilar Marín Gómez \\ Emilio García García \\ Pablo Lapunzina Badía \\ Universidad Complutense de Madrid, España Universidad Complutense de Madrid, España Hospital Universitario La Paz, España
}

\begin{abstract}
Resumen. El Síndrome de Sotos (SS) es un trastorno de crecimiento autosómico dominante, descrito por primera vez en 1.964 por Juan F. Sotos. El SS se caracteriza por un acelerado crecimiento prenatal y posnatal. Este rápido desarrollo a menudo se acompaña de retrasos y dificultades en el área motora, del lenguaje, social y cognitivo. Además, cursa con una serie de rasgos físicos, la apariencia facial parece ser el rasgo de mayor relevancia. El SS está originado por una haploinsuficiencia en el gen NSD1.

Con esta investigación pretendemos señalar las necesidades educativas más frecuentes en la población de SS y proponemos unas directrices/pautas de atención temprana ajustadas a las necesidades que suelen presentar este tipo de población. Para poder realizar esta investigación hemos seleccionado a 22 sujetos diagnosticados SS, demandado a sus familiares que nos cumplimenten un cuestionario, elaborado ad hoc, de recogida de información sobre su desarrollo y evolución.

Palabras Clave: Gen NSD1, área motora, área del lenguaje, área social, área cognitiva, dificultades de aprendizaje y atención temprana.
\end{abstract}

\begin{abstract}
Sotos Syndrome (SS) is an autosomal dominant growth disorder, described for the first time in 1964 by John F. Sotos. SS is characterized by accelerated prenatal and postnatal growth. This fast physical development is often accompanied by delays and difficulties in motor, language, social and cognitive development. In addition, it results in a series of physical characteristics, with facial appearance seeming to be the characteristic of greatest relevance. SS is caused by a haploinsufficiency in gene NSD1.

In this investigation, we aim to indicate the most frequent educative needs of those with SS and propose directives/guidelines suitable for the early attention care needs that this group usually has. In order to be able to carry out this investigation, we selected 22 subjects diagnosed with SS, and requested that their relatives complete an ad hoc questionnaire, to collect information on the development and evolution of this syndrome.

Keywords: Gene NSD1, motor area, speech area, social area, cognitive area, learning difficulties and early intervention.
\end{abstract}

\section{Introducción}

El SS es un trastorno de crecimiento autosómico dominante descrito por primera vez en 1964 por Juan F. Sotos, define este cuadro clínico como "gigantismo cerebral" (término en desuso) en referencia a la cabeza de gran tamaño típica de los niños

La correspondencia sobre este artículo debe enviarse a la primera autora a la c/ Fromista, 15 Portal D, $6^{\circ}$ A. 2850 Madrid E-mail: pi_marin@hotmail.com de SS. Este síndrome se caracteriza por un acelerado crecimiento prenatal y posnatal más notable en los primeros cuatro años de vida, situándose, frecuentemente, la talla y el perímetro cefálico en torno a los 90-97 Percentiles (Pc). Afortunadamente en la preadolescencia la diferencia comienza a cerrarse.

Este síndrome cursa con una serie de características físicas y psicológicas. La apariencia facial es la característica más clara del SS, incluye unos rasgos 
craneofaciales como: macrocefalia, dolicocefalia, frente prominente y abombada, hipertelorismo aparente, fisuras palpebrales, nariz antevertida, mejillas y nariz sonrojadas, paladar ojival, puente nasal plano, sensación de calvicie frontoparietal, barbilla puntiaguda y pabellones auriculares grandes. Manos y pies desproporcionadamente grandes en relación con su cuerpo, siendo los pies planos o colapsados hacia dentro.

En el periodo neonatal es muy frecuente la hipotonía, la ictericia prolongada y las dificultades de alimentación, hallazgos presentes en el $70 \%$ de los pacientes (Tatton-Brown et al., 2005). En edades tempranas suelen ser usuales las infecciones respiratorias superiores e infecciones de orina. Alrededor del $15 \%$ de los casos tienen problemas urinarios, especialmente el reflujo vesicouretral (Cole y Hughes, 1994; Tatton-Brown et al. 2005). También son frecuentes las otitis media de repetición.

En ocasiones, pueden presentar anomalías cerebrales; se observa dilatación de los ventrículos cerebrales sin hipertensión intracraneal y delgadez en el cuerpo calloso. En las resonancias magnéticas se ha demostrado un alargamiento y dilatación de los ventrículos, hipoplasia del cuerpo calloso, persistencia del cavum septum pellucidum y cavum vergae y macrocisterna magna"(Chen et al., 2002). Según del Valle Domínguez (2008) suelen encontrarse anomalías intracraneales obtenidas por tomografía computarizada o resonancia magnéticas, como dilatación ventricular, aumento de espacios subaracnoides y /o hipoplasia del cuerpo calloso.

Muchos pacientes de SS presentan problemas de curvatura en la columna, existiendo una gran variabilidad en cuanto a su severidad. También, algunos pacientes de SS tienen una edad ósea avanzada, es decir, por encima de su edad cronológica. Este síndrome también puede cursar con anomalías cardiacas, se observan distintas patologías en la población de SS: comunicación interauricular e interventricular, atresia o estenosis pulmonar y tricuspídea, taquicardia supraventricular, etc. (Opitz, Weaver y Reynolds, 1998). Igualmente, se han observado anomalías oftalmológicas en algunos sujetos de SS. Por último, creemos necesario resaltar que la población que padece SS tiene una mayor predisposición a padecer tumores, cuya incidencia según los estudios publicados oscila entre el $2 \%$ y el $7 \%$ de los pacientes (Hersh, Cole, Blooms, Bertolone y Hughes, 1992).

Respecto, a su desarrollo psicológico en la mayoría de los casos se aprecia déficit intelectual, pueden presentar desde graves problemas de aprendizaje hasta retraso mental, que puede variar de leve a grave en el $85 \%$ de los pacientes (Ruggieri y Arberas, 2003). Unido a estas dificultades de aprendizaje o déficit mental se aprecia un retraso en el desarrollo motor (Rutter y Cole, 1991). Además, también manifiestan un retraso en la adquisición y desarrollo del lenguaje, estando más afectado el lenguaje expresivo que en el receptivo. También pueden presentar problemas conductuales como: agresividad, berrinches, fuertes rabietas, reacciones fóbicas, rituales, ansiedad de separación y pánico ante nuevas situaciones. Estas conductas pueden venir generadas por una falta de integración y socialización, más las consecuencias de las frustraciones generadas por sus problemas intelectuales o sus dificultades de lenguaje y comunicación (Cole et al., 1994).

La etiología del SS es genética, debido a un gen dominante, concretamente "el gen NSD1, localizado en la banda q35 del cromosoma 5 (Kurotaki et al., 2002). En este gen se produce una haploinsuficiencia (anomalía genética - aberración genética), que puede presentarse de dos maneras, bien por deleción/microdeleción (pérdida de material genético) o por mutación puntual (consiste en el intercambio de material genético).

Es necesario señalar que actualmente existen sujetos diagnosticados SS, previo diagnóstico diferencial, por sus manifestaciones clínicas, sin detectarse en su gen NSD1 anomalías genéticas, pero, se cree que puede haber un error a nivel celular que esté interfiriendo con la producción adecuada de NSD1, o puede ser que algún otro gen, aún no descubierto, también cause SS.

El SS es de trasmisión vertical, es decir, si un sujeto presenta una anomalía genética en el gen NSD1 existe un $50 \%$ de posibilidades de trasmisión a cada uno de sus progenitores. Si ninguno de los padres es portador de la anomalía genética la posibilidad de tener otro hijo con SS es muy pequeña, pero no inexistente. La mayoría de los casos de SS son 
mutaciones de novo, sus padres no eran portadores.

El SS es considerado una enfermedad rara, se desconoce su prevalencia exacta, pero se estima en 1 de cada 14.000 recién nacidos, y 1 de 2000 recién nacidos con algún tipo de síndrome, pero es uno de los trastornos de sobrecrecimiento más comunes tras el síndrome de Beckwitch-Wiedeman y el Síndrome Marfan.

El presente estudio tiene como objetivo principal identificar las necesidades educativas más frecuentes en la población de SS, desde edades muy tempranas, y orientar un programa de Atención Temprana.

\section{Método}

Al ser el SS una enfermedad rara por su baja prevalencia nos supone mucho esfuerzo conseguir la muestra, con lo cual el único criterio que tenemos para seleccionar la muestra es que este diagnosticado por un médico-genetista como sujeto de SS.

La muestra es obtenida por medio del Hospital Universitario La Paz (HULP), nos ponemos en contacto con todos los familiares/pacientes diagnosticados SS. Acceden a participar en nuestra investigación 22 sujetos. 12 varones y 10 hembras, el intervalo de edad se sitúa de 3 - 28 años, pero sólo contamos con un sujeto de 28 años, por lo que la mayoría de la muestra se encuentra en el intervalo de 3 - 15 años.

Elaboramos un cuestionario donde plasmamos cuestiones que nos interesan conocer y medir relacionadas con su desarrollo evolutivo, como:

- Presencia de hipotonía en el momento de su nacimiento.

- Edad del control cefálico.

- Edad de la adquisición de la sedestación sin ayuda.

- Edad del logro del gateo.

- Edad de adquisición de la marcha autónoma.

- Presencia de torpeza motora.

- Momento de aparición de las primeras palabras.

- Edad en las que se producen las primeras combinaciones de dos palabras.

- Dificultades más frecuentes en la adquisición y desarrollo del lenguaje oral.
- Reacciona negativamente ante cualquier cambio de rutina.

- Tipo de interacciones de la población de SS.

- Tipo de Escolarización: Escolarización-Ordinaria, Escolarización-Modalidad de Integración y Escolarización-Educación Especial.

La información que obtenemos con este cuestionario es archivada en una base de datos para su posterior manejo y realización de gráficas. Los datos relacionados con el desarrollo motor y a la adquisición y desarrollo del lenguaje de la población de SS se compararán con los datos de la población normal (PN). Para conocer el momento de adquisición de cada logro motor en la población normal nos guiaremos por Palacios y Mora (2002) reflejan todos los hitos motores que se producen en los dos primeros años de vida; se mantiene sentado sin apoyo (5-9 meses), gateo (6-11 meses), agarrándose a algo puede ponerse de pie (6-12 meses) y camina por sí solo (9-17 meses). Decidimos reflejar en las gráficas en referencia a la PN el último mes de cada intervalo.

Respecto, a los datos relacionados con la adquisición y desarrollo del lenguaje oral seguimos a Carranza, Escudero y Brito (1991), los niños entre los 10 y 14 meses comienzan a producir sus primeras palabras y las primeras combinaciones de palabras aparecen entre los 18 y 24 meses. Al igual, que en las gráficas relacionadas con el área motora, en las gráficas relacionadas con el área del lenguaje también establecemos para la PN el último mes de cada uno intervalo.

\section{Resultados}

\section{Presencia de hipotonía en el momento de su nacimiento}

Entendemos por el término de hipotonía una disminución del tono muscular, también se conoce como flaccidez. La hipotonía conlleva retrasos en el desarrollo motor.

La hipotonía está presente en el momento del nacimiento en el $91 \%$ de los casos, en un $8 \%$ no se aprecia en esta etapa, aunque según señalan los padres a medida que el bebe crece se hace patente. 


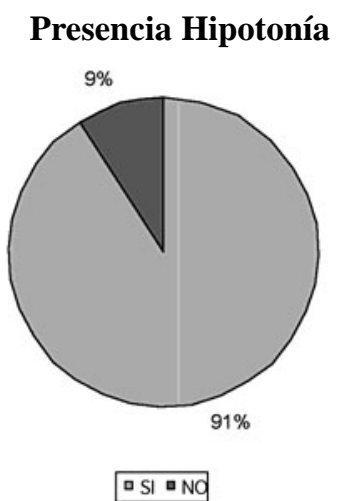

En algunos casos se acompaña esta hipotonía de cierta rigidez, pero en un número no significativo.

\section{Edad del control cefálico}

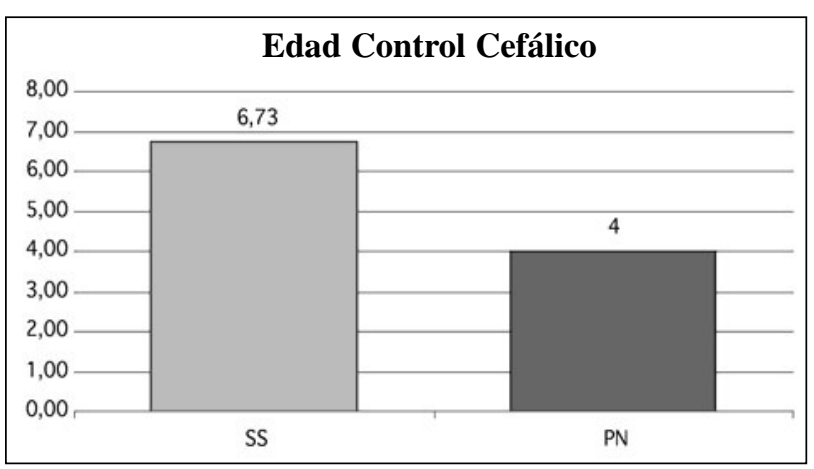

Existe un retraso bastante significativo en la adquisición del control cefálico en la población de SS, lográndose en torno a los 6,73 meses, frente a los 4 meses que es lo considerado normal.

\section{Edad de la adquisición de la sedestación sin ayuda}

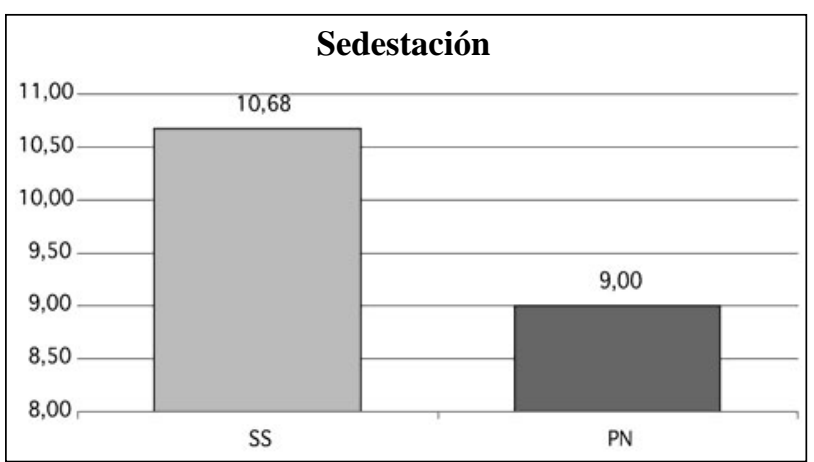

La sedestación también se adquiere con retraso en la población de SS, lográndose a los 10,68 meses, observándose un retraso si comparamos con lo establecido como normal que indica que se logrará de 59 meses.

\section{Edad de adquisición del gateo}

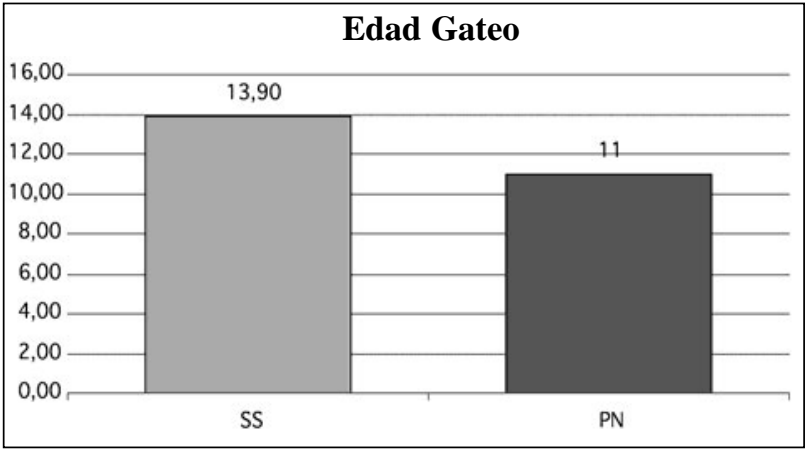

La adquisición del gateo también es susceptible de retraso, consiguiéndose alrededor de los 13,90 meses, en referencia al intervalo considerado como normal de 6-11 meses. Pero este dato debe ser manejado con mucha prudencia, ya que de los 22 sujetos evaluados solo 10 adquieren el gateo, siendo el gateo es una etapa por la que no pasan todos los niños.

\section{Edad de adquisición la marcha autónoma}

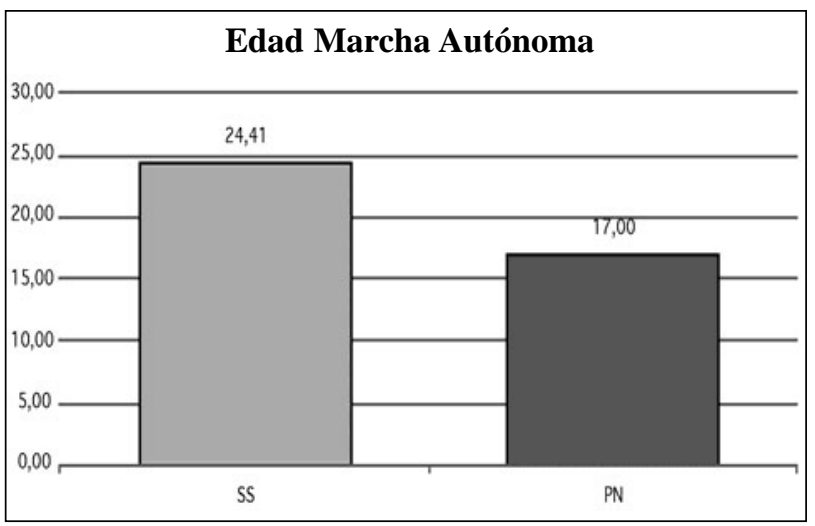

Como es de suponer si todos los logros motores valorados hasta el momento sufren retraso la marcha autónoma también sufrirá retraso, y efectivamente esto es así. La población de SS consigue la marcha sin apoyo a los 24,41 meses. Observándose un retraso bastante amplio en referencia a lo estandarizado, estableciendo que la marcha con carácter normal se podrá adquirir de lo 12-17 meses. Considerándose la ausen- 
cia de la marcha autónoma de los 16-18 meses como un signo de alerta en el desarrollo motor (Póo, 2006).

\section{Presencia de torpeza motora}

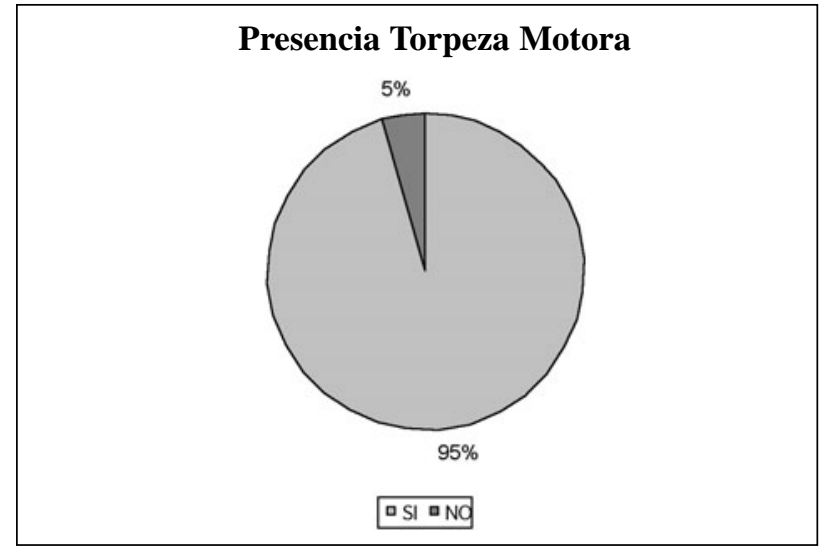

De los datos expuestos anteriormente relacionados con el desarrollo motor se confirma el retraso motor que tiene la población de SS. Este retraso motor va acompañado de cierta torpeza motora. Nosotros hemos querido evaluar en qué medida está presente la torpeza motora en la población de SS, hemos obtenido que el $95 \%$ de los sujetos de SS presenten torpeza motora.

\section{Momento de aparición de las primeras palabras}

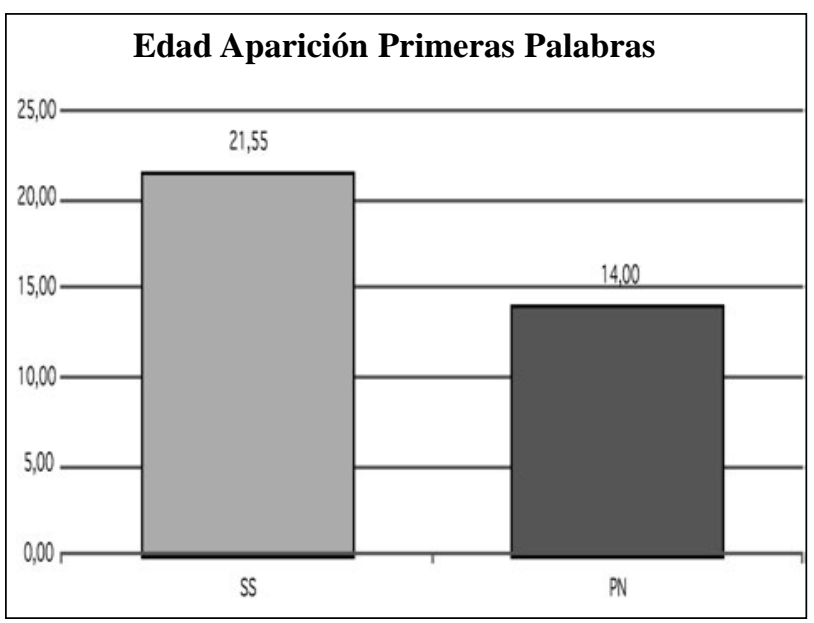

Las primeras palabras en la población normal suelen surgir alrededor de los 10-14 meses. En la población de SS aparecen a los 21,55 meses, lo que sugiere un gran retraso.
Edad en las que se producen las primeras combinaciones de dos palabras

Primeras Combinaciones de Dos Palabras

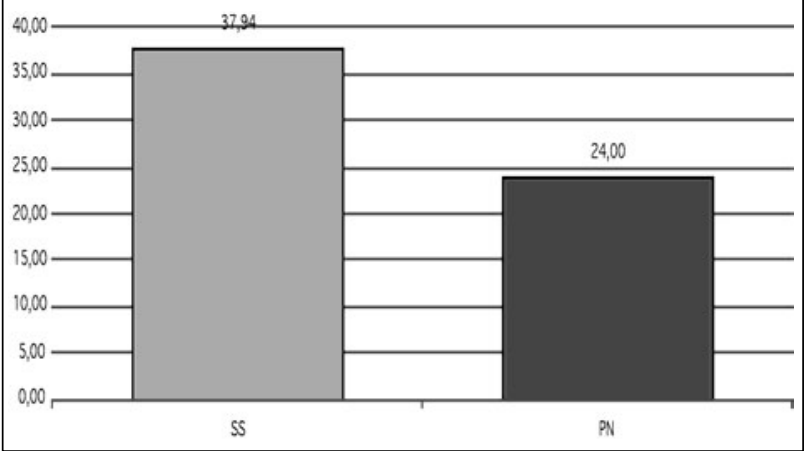

Con el concepto de primeras combinaciones de dos palabras nos estamos refiriendo a la unión de dos palabras sin flexiones ni palabras funcionales. Esto suele sucede alrededor de los 18-24 meses, si tenemos en cuenta esta referencia observamos el gran retraso existente en la población de SS ya que se producen a los 37,94 meses.

\section{Dificultades más frecuentes en la adquisición $y$ desarrollo del lenguaje oral}

Exponemos las dificultades teniendo en cuenta lo expresado por los familiares. Hemos decidido agrupar estas dificultades teniendo en cuenta los aspectos suprasegmentales del habla y las dimensiones del lenguaje (fonética-fonología, semántica, morfosintaxis, y pragmática), para una mayor claridad expositiva:

- Aspectos suprasegmentales del habla:

- Calidad vocal ronca y nasal.

- Tono monótono.

- Volumen bajo.

- Fonética.Fonología:

- Errores de articulación y pronunciación en los diferentes fonemas.

- Omisiones, no se pronuncia un sonido inicial (esa por mesa), medio (peota por pelota) o final (ma por más) o el grupo entero (ato por plato).

- Simplificación en sílabas trabadas o sinfones (consonante-consonante- vocal), como por ejemplo: "pato" por "plato".

- Sustituciones de un fonema por otro, por ejemplo: /b/ por /d/; /d/ por /t/, etc. 
- Errores en su discriminación auditiva.

- Dificultades en la secuenciación fonológica.

- Dificultades en el soplo y en coordinación fonorespiratoria.

- Dificultades en la realización de las praxias bucofaciales.

- Semántica - Léxico:

- Su vocabulario es muy reducido, pero es mucho más limitado a nivel expresivo que al nivel comprensivo.

- Morfosintaxis:

- Sus frases están formadas por un número de elementos muy reducidos, siendo estas muy sencillas.

- Dificultades para establecer la correcta coordinación gramatical entre los elementos que componen sus frases (género, número, tiempo verbal, etc.).

- Pragmática:

- Dificultades para hacer y manejar un uso correcto de las normas de comunicación.

En general los padres nos han señalado que el lenguaje comprensivo está más avanzado y con menores dificultades que el lenguaje expresivo. Algunos padres, aunque una minoría, nos han indicado que sus hijos presentaban un lenguaje ecolálico. De los 22 sujetos estudiados 5 sujetos requieren un Sistema Aumentativo de Comunicación (S.A.C.), es decir, un $22,72 \%$.

\section{Reacciona negativamente ante cualquier cambio de rutina}

Reacciona Negativamente Cambio Rutina

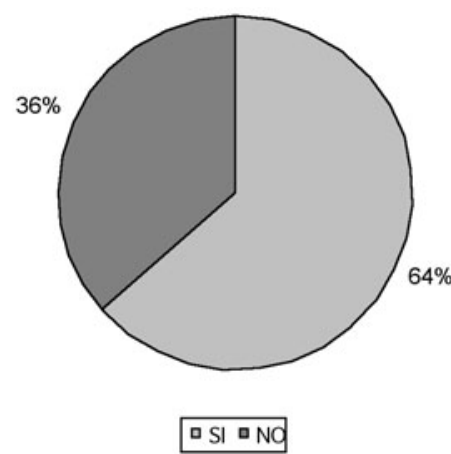

El 64\% de la población de SS reacciona negativa- mente ante cualquier cambio de rutina. Además, algunos padres nos han comunicado las manías y obsesiones que sus hijos manifiestan por determinados hechos y objetos.

\section{Tipo de interacciones de la población de SS}

Los familiares de los niños SS nos indican que cuando son pequeños tienen tendencia a relacionarse con niños más pequeños o con adultos, según van creciendo aumentan las interacciones con sus iguales, aunque se caracterizan por ser reducidas. En la adolescencia desean ser miembros de un grupo de iguales y en ocasiones esto sucede, pero en otras ocasiones son rechazados o tienen dificultades para mantener una relación con iguales por presentar diferentes intereses, etc.

Modo de Escolarización: EscolarizaciónOrdinaria, Escolarización-Modalidad de Integración y Escolarización-Educación Especial

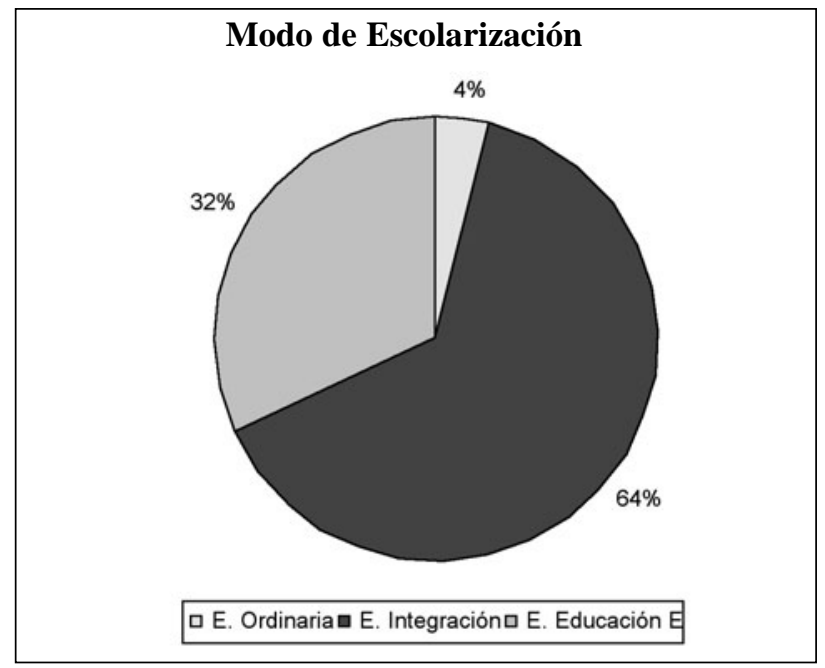

Teniendo en cuenta el modo de escolarización en la población de SS podremos deducir si existe un posible déficit cognitivo. Entendemos que sí un alumno está escolarizado en un modelo ordinario no tendrá déficit cognitivo, aunque sí posibles dificultades de aprendizaje. Pero, si se encuentra en un modo de escolarización de integración será catalogado como alumno con necesidades educativas especiales (n.e.e.), y si además precisa de adaptaciones curricu- 
lares significativas es muy probable que presente déficit cognitivo, pero este déficit siempre será menor que si se encuentra escolarizado en un modelo de Educación Especial.

Los datos estadísticos analizados sobre el tipo de escolarización de la población de SS nos indican que el $64 \%$ presenta un modo de escolarización de integración (todos ellos precisan adaptaciones curriculares significativas), el 32\% una escolarización de E.E. y el $4 \%$ una escolarización ordinaria. De esta información se desprende que el $96 \%$ de la población de SS presenta un déficit cognitivo que puede oscilar de ligero a severo. Un 4\% de la población de SS no presenta déficit cognitivo pero sí dificultades de aprendizaje, según información obtenida por sus familiares.

\section{Resultados}

La información obtenida, manejada y valorada nos arroja que estos sujetos presentan necesidades educativas en las siguientes áreas:

- Área cognitiva.

- Área motora.

- Área comunicativa-lingúística.

- Área Social.

A lo largo de esta investigación hemos ido constatando el retraso y las dificultades que la población de SS presenta en la adquisición y desarrollo del área motora y del área del lenguaje. También, gracias, al modo de escolarización y del uso de las adaptaciones curriculares significativas hemos podido conocer la incidencia de los posibles déficits cognitivos y de las dificultades de aprendizaje. Igualmente, hemos observado que un $64 \%$ reaccionan negativamente ante un cambio de su rutina habitual, y que en ocasiones, tienden a desarrollar manías, fobias, obsesiones, etc., por tanto, en el área social pueden presentar necesidades educativas.

\section{Orientaciones en su programa de Atención Temprana}

Consideramos muy importante y necesaria la atención temprana, en la población de riesgo como son los sujetos de SS, por los grandes beneficios que nos proporciona la neuroplasticidad cerebral. Como señalan Mulas y Hernández (2004), la plasticidad cerebral es el principio de organización que fundamenta la aplicación de programas de intervención terapéutica para la recuperación funcional. La plasticidad de las estructuras nerviosas es un hecho evidente y es la base teórica que respalda la intervención con programas de atención temprana.

En esta línea se sitúa Rodríguez Santos (2009) la neurociencia tienen una serie de implicaciones en los procesos educativos, la primera de ellas es considerar el aprendizaje como un factor más en la potenciación de la plasticidad neuronal, en segundo lugar, la constatación de la estrecha relación que existe entre el cerebro y el propio cuerpo proporcionando una interacción constante de los procesos cognitivos y emocionales, y en tercer lugar, la importancia de considerar los mecanismos cerebrales del aprendizaje asociativo y no asociativo, así como de los distintos sistemas de memoria. La presencia de factores genéticos y no genéticos en el desarrollo de las unidades funcionales cognitivo-neuronales a lo largo de toda la vida, abre un amplio abanico de oportunidades para el aprendizaje más allá de la etapa escolar.

Señalada la importancia que tiene la Atención Temprana pasamos a reflejar una serie de orientaciones aplicables a la población de SS. Estas orientaciones/objetivos en su momento de aplicación se ajustarán a las características, contexto y necesidades educativas propias de cada sujeto. Además, la puesta en marcha de cualquier programa de Atención Temprana requiere de forma necesaria e imprescindible de una coordinación interdisciplinar e interinstitucional.

Respecto al área motora si tenemos en cuenta la hipotonía, retraso y dificultades que presentan es conveniente que se comience a trabajar los más temprano posible y este a cargo primordialmente de un fisioterapeuta. En cuanto al desarrollo del lenguaje, tal y como hemos investigado sus dificultades, primordialmente, se centran en las cuatro dimensiones del lenguaje (fonética/fonología, semántica, morfosintaxis y pragmática), además, generalmente la comprensión está por encima de la expresión, esta área deberá estar a cargo de un logopeda. Junto a estas dos áreas se trabajará la estimulación cognitiva, a cargo de un psicólogo. Las otras necesidades educativas se trabajaran en la medida que se obser- 
ve que se están produciendo y se convierte en una necesidad educativa. Creemos necesario trabajar desde el primer momento de forma global en todas las áreas trabajadas la autonomía, autoestima y el autoconcepto. Es muy importante que la intervención empiece dando información, apoyo y asesoramiento a las familias, con el objetivo de implicarlas en el programa de actuación que se proponga.

Dado que es imposible abordar todas las áreas donde requieren intervención, nos centraremos de forma más amplia y exhaustiva en el área motora y del lenguaje.

\section{Área motora}

Una vez que se produzca el diagnóstico de SS o los familiares observen un retraso en el desarrollo motor es imprescindible comenzar la intervención fisioterapéutica. Nos parece importante señalar que esta intervención fisioterapéutica está relacionada con todas las áreas que se trabajan en el programa de Atención Temprana, pero especialmente con el área comunicativa-lingüística, ya que la psicomotricidad y el aumento del tono muscular de los órganos fonorticularios, como también la postura, etc., son aspectos que se trabajan desde la intervención fisioterapéutica y desde la intervención logopédica.

Dada que nosotros no somos profesionales del mundo de la fisioterapia únicamente nos vamos a limitar a señalar, siguiendo a Candel (2.005) una serie de objetivos para estimular las habilidades de motricidad gruesa hasta los dos años:

- De 0 a 3 meses:

- Estimulación de reflejos.

- Favorecer los movimientos del cuerpo.

- Control cefálico en diferentes posiciones.

- Estimulación vestibular.

- Volteos.

- De 3 a 6 meses:

- Reforzamiento del control cefálico.

- Control del tronco.

- Apoyo en antebrazos de prono.

- Iniciación en desplazamientos en prono.

- Apoyo en manos.

- Volteos.

- Reacciones de equilibrio.
- Toma de peso en pies.

- Sedestación: inicio.

- De 6 a 9 meses:

- Posición de rodillas.

- Control del tronco.

- Sedestación con apoyo.

- Toma de peso en manos.

- Rastreo.

- Reaccione de equilibrio.

- Sedestación sin apoyos.

- Equilibrio en sedestación.

- Mantenimiento en cuatro apoyos.

- Marcha de rodillas. Gateo.

- Cambios posturales.

- Bipedestación.

- Estimulación vestibular.

- De 9 a 12 meses:

- Reforzar adquisiciones anteriores.

- Bipedestación con apoyo.

- Equilibrio en bipedestación.

- Reacciones de equilibrio: caídas.

- Marcha con ayuda.

- Bipedestación sin apoyo.

- Estimulación vestibular.

- Marcha sin apoyo.

- De 12 a 18 meses:

- Perfeccionar los cambios posturales.

- Perfeccionamiento de la marcha.

- Equilibrio y coordinación general.

- Subir y bajar escaleras.

- Estimulación vestibular.

- Marcha rápida.

- De 18 a 24 meses:

- Reforzar y perfeccionar adquisiciones.

- Control de movimientos. Saltos.

- Carrera.

- Patear objetos.

- Coordinación dinámica general.

Estos objetivos están organizados según los hitos evolutivos del desarrollo motor normalizado, con lo cual en un niño con problemas motores como es el niño de SS necesitará, en general, más tiempo para la adquisición de cada logro motor, aunque sí seguirán las mismas etapas evolutivas, tal y como se ha mostrado en nuestra investigación. A lo largo de este periodo y a partir de los 24 meses se deberá hacer especial incidencia en el desarrollo de la psicomotri- 
cidad fina. Algunos de los objetivos de los objetivos de estimulación de habilidades motoras finas son:

- Coger objetos con ambas manos.

- Juntar las manos en línea media moviéndolas.

- Manipular objetos con ambas manos y con los dedos.

- Pasar objetos de una mano a otra.

- Etc.

\section{Área comunicativa-lingüística}

Para abordar correctamente este área es necesario conocer las etapas cronológicas que se producen en el proceso de adquisición y desarrollo del lenguaje y los logros que se alcanzan en dichas etapas; etapa prelingúística (de 0 meses hasta 12-15 meses) y etapa lingüística (de 1 año hasta los 7 años aproximadamente), por motivos de espacio no vamos a proceder a explicarlas de manera exhaustiva. Por tanto, el desarrollo evolutivo lingüístico nos servirá de guía para poder establecer los objetivos a trabajar, teniendo como referencia las necesidades educativas lingüísticas que manifiesta la población de SS. Planteamos una intervención ajustada a la gran mayoría de dificultades del lenguaje que presentan los niños de SS, pero es necesario señalar tal y como hemos indicado anteriormente que existen sujetos que precisan de un S.A.C., pero dado que es una minoría nos centramos en la intervención de la mayoría de las dificultades que hemos comprobado.

A continuación, señalamos una serie de objetivos a trabajar en la etapa prelingüística:

- Estimular verbal y táctilmente al niño.

- Conseguir la orientación a estímulos visuales y auditivos.

- Lograr el contacto ocular y la fijación de la mirada.

- Estimular y adquirir la sonrisa social.

- Fomentar y establecer las pautas de intervención.

- Conseguir una adaptación social y afectiva.

- Favorecer y lograr la imitación.

- Responder activamente a indicaciones sencillas: "no", "dame", "adiós"...

- Comprender la prohibición.
- Iniciar la comprensión y expresión verbal.

A parte de estos objetivos, en ocasiones, tenemos que priorizar el siguiente:

- Aprender a deglutir y tragar.

En la medida que realice una correcta deglución y consiga tragar se facilitará el logro de los objetivos que planteamos en la etapa prelingüistica y lingüística. La intervención fisioterapéutica también contribuye a la consecución de dicho objetivo, de ahí la importancia de la estrecha colaboración entre el logopeda y el fisioterapeuta, que anteriormente expusimos.

En la etapa lingüística proponemos trabajar objetivos relacionado con los prerrequisitos del lenguaje y con las dimensiones de este. Algunos aspectos a trabajar dentro de los prerrequisitos del lenguaje son:

- Fomentar la intención comunicativa.

- Desarrollar la capacidad memorística.

- Mantener y aumentar el contacto ocular.

- Favorecer el desarrollo del juego simbólico.

- Desarrollar los turnos mediante juegos.

Respecto a las dimensiones del lenguaje hemos decidido señalar los objetivos a trabajar y posibles actividades para la consecución de estos. A continuación exponemos ejemplos, para una mayor claridad expositiva, de cómo se desarrollarán determinadas actividades que pertenecen a la dimensión pragmática y que pretenden alcanzar el objetivo de desarrollar un lenguaje social.

Para la petición de objetos señalamos los pasos a seguir:

- Hacer posible que el niño vea el objeto deseado.

- Nombrar en presencia del niño el objeto deseado.

- Presentar al niño el índice natural previo a la aparición del evento deseado.

- Le proporcionamos la siguiente consigna: "Yo quiero..." "Dame".

Ejemplos:

- Nos quedamos rezagados a la hora de trabajar sin proporcionarle (lápiz, tijeras, papel, pegamento, piezas para un juego...).

- Ponerle el desayuno en el centro de la mensa y no puede coger los alimentos hasta que no se los pida al adulto (desayuno de material logopédico). 


\section{FONÉTICA-FONOLOGIA}

\section{OBJETIVOS}

Desarrollar la capacidad básica para percibir e interpretar sonidos, emisiones del habla y captar las estructura del lenguaje.

\section{ACTIVIDADES}

Discriminar auditivamente: sonidos de la casa, corporales, instrumentos musicales, de la naturaleza...

Discriminación de tonos graves-agudos.

Ejercicios de intensidad que son alta y baja.

Asociación de sonidos.

Secuencias auditivas.

Reconocer las sílabas aisladas.

Reconocer sílabas dentro de una palabra.

Conseguir un buen control del soplo y desarrollar la coordinación costo-diafragmática.

Inspiración nasal/bucal (rápido-lento- distintos tiempos).

Espiración nasal/bucal (rápido-lento- distintos tiempos).

Ejercicios de inspiración/espiración.

Expulsión del aire emitiendo un sonido vocálico o una espiración silbante (con apoyo visual de dibujos: un camino, una serpiente...)

Ejercicios para mejorar el soplo como por ejemplo: inflar globos, soplar velar, utilización de matasuegras...

Ejercicios de relajación utilizando el Método Schultz o el Método Jacobson.

Aumentar el control de la tonicidad y coordinación de los músculos de la articulación.
Ejercicios de praxias: de labios, de lengua (externos e internos), faciales, del velo del paladar, de mandíbula y de dientes.
Adquirir el patrón articulatorio de los fonemas en sus distintas posiciones (sílaba directa-sílaba indirecta- sílaba mixta y sílaba trabada), expresándose oralmente con una articulación adecuada y comprensible.
Ejercicios de posición de los órganos fonoarticulatorios.

Ejercicios de emisión aislada de un fonema.

Repetición de sílabas, palabras, frases, versos...

Memorizar y recitar adivinanzas, trabalenguas...

\section{MORFOSINTAXIS}

\section{OBJETIVOS}

Utilizar los componentes morfológicos del lenguaje para desarrollar la capacidad de precisión comunicativa.

Desarrollar la expresión oral mediante la construcción de frases adecuadas.

\section{ACTIVIDADES}

Formación de plurales regulares e irregulares.

Formación de género.

Formación de adjetivos.

Verbos regulares en pasado-presente-futuro.

Sustantivos en aumentativo y diminutivo..

Construcción de frases de 2 elementos y progresivamente ir aumentando el número de elementos que componen las frases. En principio podremos ayudarnos de pictogramas que supongan un sujeto y una acción, posteriormente artículo, objeto...

Introducción de la negación.

Introducción del nexo "y".

Formar frases a partir de una palabra dada.

\section{LÉXICO-SEMÁNTICA}

\section{OBJETIVOS}

Desarrollar la competencia léxico (vocabulario)semántica (familia de palabras, semejanzas/diferencias, contrarios, asociación de palabras...)

\section{ACTIVIDADES}

Denominación de objetos, fotos y dibujos presentes y ausentes.

Petición de objetos presentes y ausentes.

Juegos de reconocimiento de palabras.

Aprendizaje de contrarios y semejanzas.

Definición de palabras.

Formar familias de palabras.

Descripción de láminas. 


\section{PRAGMÁTICA}

\begin{tabular}{ll}
\hline OBJETIVOS & ACTIVIDADES \\
\hline Desarrollar un uso de lenguaje social. & $\begin{array}{l}\text { Fomentar el uso de los saludos sociales. } \\
\text { Actividades que fomenten la utilización de los términos básicos de cortesía (por favor, } \\
\text { gracias - de nada...) }\end{array}$ \\
Petición de un objeto. \\
Petición de acción. \\
Actividades para conseguir la negación. \\
Solicitud de ayuda y permiso. \\
Formulación de preguntas - respuestas. \\
A continuación expondremos los pasos para conseguir la petición de un objeto, permiso \\
ayuda, realización de preguntas-respuestas, etc.
\end{tabular}

Pasos para la petición de permiso:

- Impedir de forma aleatoria que el alumno haga algo que desea.

- Consigna: ¿Puedo?

Ejemplos:

- Para ir al servicio cuando estemos en clase.

- Para tomar el desayuno.

- Para coger el juguete a la hora del recreo o jugar con algo.

- Para jugar al ordenador.

Respecto a las preguntas-respuestas fomentaremos el siguiente tipo y de la siguiente forma:

- Pregunta ¿Qué es esto? Respuesta: denominación.

- Pregunta ¿Qué quieres? Respuesta: petición.

- Pregunta ¿Cómo estás? Respuesta: expresar sentimientos.

- Pregunta: ¿Quieres...? Respuesta: dar información o rechazo o deseo.

- Pregunta: ¿Está (persona)? Respuesta: dar información.

- Pregunta: ¿Quién quiere (objeto/acción)? Respuesta expresar deseo.

- Pregunta: ¿Cómo es (persona)? Respuesta: expresar cualidades.

\section{Conclusiones y limitaciones del estudio}

A lo largo de esta investigación hemos comprobados como los sujetos que padecen SS presentan necesidades educativas relacionadas con diversas áreas del desarrollo. Por ello, hemos resaltado la importancia y necesidad de implantar de forma sistémica y lo precoz posible un programa de Atención Temprana ajustado a las necesidades y características de cada sujeto.

Después de finalizar esta investigación hemos conocido que 2 sujetos de 4 y 5 años respectivamente, que comenzaron a recibir Atención Temprana a los 3 y 4 meses actualmente no requieren adaptaciones curriculares significativas, por lo que consideramos que este hecho vuelve a confirmar la neuroplasticidad cerebral y la justificación e importancia de la Atención Temprana.

A lo largo de esta investigación nos hemos encontrado con numerosas dificultades. Encontrar un número de sujetos considerable que conformaran la muestra nos supuso grandes dificultades, ya que el SS es considerada una enfermedad rara por su baja prevalencia. Por otro lado, decidimos elaborar un cuestionario y solicitar a las familias su cumplimentación y la posibilidad de acceder a determinados informes (médicos, psicológicos, fisioterapéuticos, etc.), para conocer sus características y desarrollo evolutivo. Optamos por este tipo de instrumento por la dificultad de contactar físicamente con la gran mayoría por su ubicación geográfica. Consideramos que además de la utilización de este tipo de instrumento hubiera sido necesario la realización de una evaluación psicopedagógica y un seguimiento directo de cada uno de ellos. Proponemos una investigación futura centrándonos en dos sujetos a los cuales podamos realizar una evaluación psicopedagógica y un seguimiento directo. 


\section{Extended Summary}

Sotos syndrome (SS) is a autosomal dominant growth disorder, described for the first time in 1964 by John F. Sotos. SS is characterized by accelerated prenatal and postnatal growth. This fast physical development is often accompanied by delays and difficulties in motor, language, social and cognitive development. In addition, it results in a series of physical characteristics, with facial appearance seeming to be the characteristic of greatest relevance. SS is caused by a haploinsufficiency in gene NSD1.

The intended aim of this investigation is to identify the educative needs that SS sufferers most often have. To this end, we selected 22 SS-diagnosed subjects, all diagnosed by an SS-geneticist doctor. We drew up a questionnaire in which we included questions related to the evolution of the syndrome, and which made reference to its different development areas. We observe that all the SS subjects show difficulty and delay in the motor area. The same applies to the communicative-linguistic area, with receptive language being more affected than expressive language. $96 \%$ show some type of cognitive deficit, oscillating from mild to severe. Also, we have seen that difficulties may be present in the social area, with $64 \%$ having negative feedback when faced with changes of routine, and some developing odd habits, phobias, obsessions, and so on.

We propose paths for the development of an Early Attention program suited to the educative needs most frequently shown in the SS population. The need to propose an Early Attention program is justified by the neuroplasticity principle. Our guidelines are characterized by a systemic approach and require interdisciplinary and inter-institutional coordination. We concentrated particularly on the motor and the communicative-linguistic area, without forgetting cognitive stimulation and working, in all interventions, on autonomy, self-esteem and selfconcept. With regard to the social area, prevention will be established to avoid certain needs arising, but if they were to appear, working on the change in routine is recommended by means of diaries with pictograms.

We will concentrate, especially for reasons for space, on the communicative-linguistic area. Once the diagnosis of SS takes place, or the relatives observe a delay in motor development, it is essential to start with the physiotherapy intervention. It seems important to us to mention that this physiotherapy intervention is related to all areas in the Early Attention program, but especially to the communicative-linguistic area, since the motor skills and the increase of muscular tone in the fonorticularios organs, as well as the position, etc., are aspects that are worked on in physiotherapy and speech therapy interventions.

Since we are not therapists, we will follow Candel (2005) in the establishment of the possible objectives.

- From 0 to 3 months:

- Stimulation of reflexes.

- To encourage movement of the body.

- Cephalic control in different positions.

- Vestibular stimulation.

- Turning over.

- From 3 to 6 months:

- Reenforcement of cephalic control.

- Control of the trunk.

- Use of forearms when in prone position.

- Start of movement from position prone.

- Support in hands.

- Turning over.

- Reactions of balance.

- Taking of weight on feet.

- Starting to learn to sit.

- From 6 to 9 months:

- Kneeling position.

- Control of the trunk.

- Seating support.

- Taking weight in hands.

- Tracking.

- Balance reactions.

- Seating without support.

- Balance in sitting.

- Maintenance of position on all fours.

- Moving on knees. Crawling.

- Changes of position.

- Standing.

- Vestibular stimulation. 
- From 9 to 12 months:

- Reinforcement of previous development.

- Standing on two feet with support.

- Balance when standing on two feet.

- Balance reactions: falls.

- Walking with aid.

- Standing on two feet without support.

- Vestibular stimulation.

- Walking without support.

- From 12 to 18 months:

- Improving changes in posture.

- Improvement of walking.

- Balance and general coordination.

- To go up and down stairs.

- Vestibular stimulation.

- Fast walking.

- From 18 to 24 months:

- To reinforce and to perfect development so far.

- Control of movements. Jumps.

- To race.

- To kick objects.

\section{General dynamic coordination}

These objectives are organized according to the evolutionary landmarks of standard motor development. Therefore, a child with motor problems, such as those of a SS child, will need, generally, more time for the acquisition of each motor achievement, although they will follow the same developmental stages, as we have seen in our investigation. Throughout this period, and from 24 months, special emphasis must be given to the development of fine motor skills. Some objectives of the promotion of fine motor skills are:

- To take objects with both hands.

- To join the hands in midline moving them.

- To manipulate objects with both hands and the fingers.

- To pass objects from one hand to another one.

- Etc.

Regarding the communicative-linguistic area, we make a necessary distinction between the prelinguistic stage and the linguistic stage. We propose an intervention tailored to the majority of language dif- ficulties present in children with SS, but it is important to indicate that there are also children who require an alternative system of communication. Some of the objectives that we establish in the prelinguistic stage are:

- Verbal and tactile stimulate of the child.

- To achieve orientation towards visual and auditory stimulii.

- To make and matain eye contact.

- To encourage and acquire the social smile.

- To promote and establish guidelines for intervention.

- To secure social and emotional adjustment.

- To promote and achieve imitation.

- To respond actively to follow simple indications actively: "no", "give it to me", "goodbye" ...

- To understand prohibition.

- To initiate understanding and expression.

- In addition to these goals, sometimes we must prioritize the following:

- Learn to swallow.

The ability to swallow correctly will facilitate the objectives raised in the Pre-linguistic and linguistics stages. Physiotherapeutic intervention also contributes to the achievement of this objective, thus the importance of close collaboration between the speech therapist and the physiotherapist, that we previously exposed.

In the linguistic stage, we propose working on objectives related to the prerequisites of the language and the dimensions of this. Prerequisite of the language:

- To promote the communicative intention.

- To develop the ability of memory.

- To maintain and enhance eye contact.

- To favor the development of symbolic play.

- Develop turn-taking through games.

- Finally, we will expose some of the objectives to work on in the four dimensions of language:

- Phonetics / Phonology

- To develop the basic ability to perceive and interpret sounds, utterances and capture the structure of language.

- To achieve good breath control and develop ribcage-diaphragmatic coordination. 
- To increase the control of muscle tone and coordination of the muscles of articulation.

- To acquire the articulatory pattern of phonemes in different positions (direct-syllable, indirect syllable, mixed syllable and joined syllable), expressing orally with suitable and comprehensible articulation.

- To use the morphological components of the language to develop the ability of communicative precision.

- Morphosyntaxis

- To use the morphological components of the language to develop the ability to communicative with precision.

- To develop oral expression by means of the construction of suitable phrases.

- Lexical-Semantic

- To develop lexical (vocabulary) and semantic (family of words, similarities/differences, opposites, association of words) competence.

- To use the morphological components of the language to develop the ability to communicate accurately.

- To develop oral expression through appropriate sentence construction.

- Pragmatics

- To develop a use of social language.

We consider that an early implementation of the Early Childhood program with a systemic approach will significantly improve prognosis, enabling the person with SS to achieve greater autonomy and possible independence.

\section{Referencias}

Agwu, J. C., Shaw, N. J., KirK, J., Champman, S., Ravine D. y Cole, T. R. (1999). Growth in Sotos syndrome. Archives of Disease in Childhood, 80 , 339-342.

Bisetto Pons, D. y Latorre Latorre, A. (2009). Trastornos del desarrollo motor. Madrid: Pirámide.

Boer, L., Kant, S., Karperien, M., Tjon, J., Vink, G., Dauwerse, H., ... y Beemer. F. (2004). Genotype-
Phenotype correlation in patients suspected of having Sotos syndrome. Hormone Research in Paediatrics, 62, 197-207.

Boer, L., Röder, I. y Wit., J. M. (2006). Psychosocial, cognitive and motor functioning in patients with suspected Sotos syndrome: a comparison between patients with and without NSD1 gene alterations. Developmental Medicine and Chid Neurology, 48, 582-588.

Candel Gil, I. (2005). Elaboración de un programa de atención temprana. Revista Electrónica de Investigación Psicoeducativa, 3, 151-192.

Carranza, J. A., Escudero, A. y Brito, A. G. (1991). De las palabras aisladas a las combinaciones de palabras. Anales de psicologia, 7, 161-180.

Cecconi M., Forzano F., Milani D., Cavani S., Baldo C., Selicorni A., ... Faravelli, F. (2005). Mutation analysis of the NSD1 gene in a group of 59 patients with congenital overgrowth. American Journal of Medical Genetics Part A, 134, 348-349.

Cole, T.R. (1990). Sotos syndrome. Journal o Medical Genetics, 27, 571-576.

Cole T. R. y Hughes H. E. (1994). Sotos syndrome: a study of the diagnostic criteria and natural history. Journal of Medical Genetics, 31, 20-32.

Chih-Ping, C., Shuan-Pei, L., Tung-Yao, C., NanChang C., Shin-Lin, S., Chen-Ju, L., ... HongChin, H. (2002). Perinatal imaging findings on inheridet Sotos syndrome. Prenatal Diagnosis, 22, 887-892.

Del Valle Dominguez, J. M. (2008). Caracterización molecular del Síndrome de Sotos y estudio de otras causas genéticas de hipercrecimiento. Facultad de biología. Barcelona: Universidad Pompeu Fabra.

Douglas J., Coleman K., Tatto-Brown K., Hughes H. E., Temple I. K., Cole T. R. y Rahman N. (2005). Evaluation of NSD2 and NSD3 in overgrowth syndrome. European Journal of Human Genetics, 13, 150-153.

Douglas, J., Hanks, S., Temple, I. K., Davies, S., Murray A., Upadhyaya M., ... Rahman N. (2003). NSD1 mutations are the major cause Sotos syndrome and occur in some cases of Weaver syndrome but are rare in the overgrowth phenotypes. The American Journal of Human Genetics, 72, 132-143. 
Faravelli, F. (2005). NSD1 mutations in Sotos syndrome. American Journal of Medical Genetics Part C: Seminars in Medical Genetics, 137, 24-31.

Fernández-Toral, J., Álvarez-Berciano, F., BarreiroDaviña, J., Fernández-Diego, J. I., y RodríguezPosada, R. M. Gigantismo Cerebral (Síndrome de Sotos). Oviedo: Universidad de Oviedo.

Funez, F., Talesnik, E. y Muzzo, S. (1979). Gigantismo Cerebral o Síndrome de Sotos. Revista Chilena de Pediatría, 50, 64-67.

Gusmao Melo, D., Pina-Neto, J. M. y Acosta, A. X. (2000). Neuroimaging and echocardiographic findings in Sotos síndrome. The American Journal of Human Genetics, 90, 432-484.

Gusmao, D., Xavier, A., Almeida, M. A., Monteiro, J. y Vieira de Castro, J. A. (2002). Sotos Syndrome (Cerebral Gigantism) Analysis of 8 cases. Neuropsiquiatria, 60, 234-238.

Hersh, J. H., Cole, T. R., Blooms, A. S., Bertolone, S. J., Hughes, H. E. (1992). Risk of malignancy in Sotos syndrome. Journal Pediatric, 120, 572-574.

Höglund, P., Kurotaki, N., Kytölä, S., Miyake, N., Somer, M. y Mastsumoto, N. (2003). Familial Sotos syndrome is caused by novel $1 \mathrm{bp}$ deletion of the NSD1 gene. Journal of Medical Genetics, 40, 51-54.

Imaizumi, K., Kimura, J., Matsuo, M., Kurosawa K., Masuno, M., Niikawa, N. y Kuroki ,Y. (2002). Sotos syndrome associated with a de novo balanced reciprocarl translocation $\mathrm{t}(5 ; 8)$ (q35; 24.1$)$. American Journal of Medical Genetics, 107, 5860.

Kamimura, J., Endo, Y., Kurotaki, N., Kinoshita, A., Miyake, N., Shimokawa, O., ... Matsumoto, N. (2003). Identification of eight novel NSD1 in Sotos syndrome. Journal of Medical Genetics, 40, 126.

Kurotaki, N., Harada, N., Shimokawa, O., Miyake, N., Kawame, H., Uetake, K., ... Matsumoto, N. (2002). Fifty microdeletions among 112 cases of Sotos syndrome: low copy repets possibly mediate the common deletion. Human Mutation, 22, 378-387.

Kurotaki, N., Imaizumi, K., Harada, N., Masuno, M., Kondoth, T., Nagai, T., ... Matsumoto, N. (2002). Haploinsufficiency of NSD1 causes Sotos Syndrome. Nature Genetics, 30, 365-366.
Lapunzina, P. (2005). Other tumors in Sotos Syndrome. American Journal of Medical Genetics, 135, 228.

Lapunzina, P. (2005). Risk o tumorigenesis in overgrowth syndromes: a comprehensive review. American Journal of Medical Genetics. PartC, Seminars in Medical Genetics, 137, 53-71.

Lapunzina, P., Soler, V. y Gracia, R. (2005). Riesgo tumoral en los síndromes de sobrecrecimiento. En R. Grañcia (Eds.), Endocrinología pedíatrica y del adolescente (pp. 567-567). Madrid: Gráficas Letra, S. L.

Melchior, L., Schwartz, M., Duno, M. (2005). dHPLC screening of the NSD1 gene identifies nine novel mutations-summary of the first 100 Sotos syndrome mutations. Annals of Human Genetic, 69, 222-226.

Mulas, F. y Hernández, S. (2004). Bases neurobiológicas de la atención temprana. En J. Pérez-López y A. G. Brito (Coord.), Manual de Atención Temprana (pp. 45-56). Madrid: Editorial Pirámide.

Nagai, T., Matsumoto, N., Kurotaki, N., Harada, N., Niikawa, N., Ogata, T., ... Naritomi, K. (2003). Sotos syndrome and haploinsufficiency of NSD1: clinical features of intragenic mutations and submicroscopic deletions. Journal of Medical Genetics, 40, 285-289.

Opitz, J. M. (1998). The síndromes of Sotos and Weaver: reports and review. American Journal of Medical Genetics, 79, 294-304.

Palacios, J. y Mora, J. (2002). Crecimiento físico y motor hasta los 2 años. En J. Palacios, A. Marchesi y C. Coll. Desarrollo psicológico y educación. Vol. I: Psicología Educativa y Educación. Madrid: Alianza Editorial.

Póo, P. (2006). Desarrollo psicomotor: características evolutivas de 0-3 años, signos de alerta. Manejo Terapéutico. V Reunión anual de la sociedad Asturiana de pediatría de Atención Primaria.

Puyuelo, M. (2003). Manual del desarrollo y alteraciones del lenguaje. Barcelona: Masson.

Rae Anderson, R., Buehler, B. A. y Schaefer, B. (2000). Sotos syndrome: a Handbook for families. Nebraska: University of Nebrasca Medical Center.

Res, H. (2004). Molecular Basis of Sotos Syndrome. Hormone Research, 62, 60-65.

Rio, M., Clech, L., Amiel, J., Faivre, L., Lyonnet, S., 
Le Merrer, M., ... Cormier-Daire, V. (2003). Spectrum of NSD1 mutations in Sotos and Weaver syndromes. Journal of Medical Genetics, 40, 436-40.

Rodríguez Santos, F. (2009). Educación y Neurociencia. Psicología Educativa, 5, 27-38.

Ruggieri, V. L. y Arberas C. L. (2003). Fenotipos conductuales. patrones neuropsicológicos biológicamente determinados. Revista de Neurología, 37, 239-253.

Rutter, S. C. (1991). Psychological characteristics of Sotos syndrome. Developmental Medicine and Child Neurology, 33, 898-902.

Sarimski, K. (2003). Behavioural and emotional characteristics in children with Sotos syndrome and learning disabilities. Developmental Medicine and Child Neurology, 45, 172-178.

Sobradillo, B., Aguirre, A., Aresti, U., Bilbao, C., Fernández Ramos, A., Lizárraga, A., ... Hernández, M. (2004). Curvas y Tablas de Crecimiento (Estudios Longitudinal y Transversal). Bilbao: Fundación Faustino Orbegozo Eizaguirre.

Sotos, J. F. (2004). Actualización del hipercrecimiento. Anales de Pediatría, 60 (Supl. 2), 79-86.

Sotos, J. F. y Dodge, P. R. (1964). Cerebral gigantism in childhood. New England Journal of Medicine, 271, 109-116.

Tatton-Brown, K., Douglas, J., Coleman, K., Baujat, G., Cole, T. R., Das, S., ... Childhood Overgrowth Collaboration. (2005). Genotype-Phenotype associations in Sotos syndrome: An analysis of 266 individuals with NSD1 aberrations. The American Journal of Human Genetics, 77, 193-204.

Tatton-Browm, K., Douglas, J., Coleman, K., Baujat, G., Chandler, K., Clarke, A., ... Reardon. W. (2005). Multiple mechanisms are implicated in the generation of $5 \mathrm{q} 35$ microdeletions in Sotos Syndrome. Journal of Medical Genetics, 42, 307313.
Tatton-Brown K., Douglas J., Coleman K., Baujat G., Cole T. R., Das S., ... Childhood Overgrowth Collaboration (2005). Genotype-phenotype associations in Sotos Syndrome: an analysis o 266 individual with NSD1 aberrations. Annals Journal Human Genetics, 77, 193-204.

Tatton-Brown K., Douglas J., Coleman K., Baujat G., Chandler K., Clarke A., ... Childhood Overgrowth Collaboration (2005). Multiple mechanisms are implicated in the generation of $5 \mathrm{q} 35$ microdeletions in Sotos syndrome. Journal of Medical Genetics, 42, 307-313.

Tatton-Brown, K. y Nazneen, R. (2007). Sotos Syndrome (Practical Genetics). European Journal of Human Genetics, 15, 264-271.

Tatton-Brown K., Rahman N. (2007). Sotos syndrome. European Journal of Human Genetics, 15, 264-271.

Tsukahara, M., Murakami, K., Lino, H., Tateishi, H., Fujita, K. y Uchida, M. (1999). Congenital heart defects in Sotos syndrome. American Journal of Medical Genetics, 84, 172.

Vila, I. (2002). Adquisición del lenguaje. En J. Palacios, A. Marchesi y C. Coll. Desarrollo psicológico y educación. Vol. I: Psicología Educativa. Madrid: Alianza Editorial

Visser, R., Hasegawa, T., Niikawa, N. y Matsumoto, N. (2006). Analiysis of the NSD1 promoter region in patients with a Sotos syndrome phenotype. Journal Human Genetics, 51, 15-20.

Weaver, D. D. (1994). Overgrowth syndrome and disorders: Definition, classification and discussion. Growth Genetics Hormame, 10, 1-4.

Winship, I. M. (1985). Sotos syndrome. Autosomal dominant inheritance substantiated. Clinical Genetics, 28, 243-246.

Zonana, J., Sotos, J.F., Romshe, C. A., Elders y M. J. (1977). Dominant inheritance o cerebral gigantism. Journal Pediatric, 91, 251-256.

Manuscrito recibido: 02/05/2011

Revisión recibida: 13/06/2011

Manuscrito aceptado: 16/06/2011 